\title{
Dominant Processes during Human Dendritic Cell Maturation Revealed by Integration of Proteome and Transcriptome at the Pathway Level
}

\author{
Sonja I. Buschow, ${ }^{\dagger, \ddagger}$ Edwin Lasonder, ${ }^{\ddagger, \S}$ Hanneke W. M. van Deutekom, ${ }^{\dagger, \S}$ Machteld M. Oud, ${ }^{\dagger}$ \\ Luca Beltrame," Martijn A. Huynen, ${ }^{\S}$ I. Jolanda M. de Vries, ${ }^{\dagger}$ Carl G. Figdor, ${ }^{*,+,}$ and \\ Duccio Cavalieri ${ }^{\prime \prime \perp}$
}

\begin{abstract}
Department of Tumor Immunology and CMBI at the Nijmegen Centre for Molecular Life Sciences, Radboud University Nijmegen Medical Centre, Postbox 9101, 6500 HB Nijmegen, The Netherlands, and Department of Pharmacology, University of Florence, Florence, Italy
\end{abstract}

Received September 22, 2009

\begin{abstract}
Gene expression is commonly used to study the activation of dendritic cells (DCs) to identify proteins that determine whether these cells induce an immunostimulatory or tolerogenic immune response. RNA expression, however, does not necessarily predict protein abundance and often requires large numbers of experiments for statistical significance. Proteomics provides a direct view on protein expression but is costly and time consuming. Here, we combined a comprehensive quantitative proteome and transcriptome analysis on a single batch of immature and cytokine cocktail matured human DCs and integrated resulting data sets at the pathway level. Although overall correlation between differential mRNA and protein expression was low, correlation between components of DC relevant pathways was significantly higher. Differentially expressed proteins and genes partly mapped to identical but also to different pathway components demonstrating that RNA and protein data not only supported but also complemented each other. We identified 5 dominant pathways, which confirmed the importance of cytokines, cell adhesion, and migration in DC maturation and also indicated a fundamental role for lipid metabolism. From these pathways we extracted novel maturation markers that might improve DC vaccine design. For several of the candidate markers we confirmed widespread significance examining DCs from multiple individuals, underscoring the validity of our approach. We conclude that integration of different but related data sets at the pathway level can significantly increase the predictive power of multi "omics" analyses.
\end{abstract}

Keywords: dendritic cell • proteomics • transcriptomics • pathway analysis • antigen presenting cell

\section{Introduction}

Dendritic cells (DC) are professional antigen presenting cells that link innate and adaptive immunity. ${ }^{1}$ DCs are able to identify and ingest pathogens, apoptotic or infected cells, as well as malignant cells. They degrade the ingested material and present resulting antigenic fragments via major histocompatibility complexes (MHC) to $\mathrm{T}$ cells to induce an adaptive immune response. ${ }^{2}$ Therefore, DCs are central players in the immune response and depending on their maturation and activation state they determine the final outcome of the

\footnotetext{
* To whom correspondence should be addressed. Dr. Carl Figdor, Department of Tumor Immunology, Nijmegen Centre for Molecular Life Sciences, Radboud University Nijmegen Medical Centre, Nijmegen, P.O. Box 9101, 6500 HB Nijmegen, The Netherlands. E-mail: c.figdor@ncmls.ru.nl.

${ }^{\dagger}$ Department of Tumor Immunology, Radboud University Nijmegen Medical Centre.

${ }^{\ddagger}$ These authors contributed equally to this work.

${ }^{\S}$ CMBI at the Nijmegen Centre for Molecular Life Sciences, Radboud University Nijmegen Medical Centre.

" University of Florence.

${ }^{\perp}$ These authors contributed equally to this work.
}

immune response, which can be either tolerogenic or immunogenic. ${ }^{3}$ The exact nature of the immune response is dictated by signals from the ingested material that are recognized by the DC but also by cytokines derived from other infected cells. ${ }^{4}$ Such signals drive DC maturation by altering its cytokine and surface protein expression and by triggering its subsequent migration from the periphery to the lymph node where it can interact with $\mathrm{T}$ and $\mathrm{B}$ cells. The huge and complex cellular changes that are associated with DC maturation take place in no more than $48 \mathrm{~h}$ and are determined by the integrated signals from among others cytokine- and chemokine- receptors, pathogen recognition receptors, endocytic receptors and adhesion receptors. This ensemble of signals then leads to specific DC gene and protein expression profiles.

The central role of DC in the initiation of immune responses and their ability to induce immunity as well as tolerance is currently exploited to fight certain types of cancer and infectious diseases or to prevent autoimmunity and transplant rejection respectively. ${ }^{5-7}$ Further development of these therapies however requires fundamental knowledge on factors that 
hold the crucial balance between immunity and tolerance and thus determine DC function. To this end, the DC maturation process has been studied in great detail using large scale RNA microarray chips. ${ }^{8}$ Due to lack of reproducibility and interindividual genetic variation however, large numbers of replicates are required for the discovery of predictive markers. Increasing the number of biological replicates, although still essential to assess interindividual variation, is not the only way to get more reproducible information out of large (mRNA) data sets (refs 9 and 10 and references therein). Rather, methods to extract more general information on affected processes or biological pathways may improve the comparability of data sets. ${ }^{10}$ Similarly, combining data on multiple levels (e.g., mRNA and protein) from one biological sample at the pathway level may bring forward processes that are affected the most and in this way improve the reliability of general information obtained from that sample.

It is well known that mRNA expression not necessarily reflects actual protein expression because transcripts can be translational nonactive, protein and RNA may differ in stability, or proteins are regulated by post-translational modifications rather than transcriptional ones. Proteomics, on the other hand, measures proteins directly but is more laborious and resource demanding, preventing elaborate analysis of multiple technical and/or biological replicates by most research groups. Nonetheless, several groups have attempted to study changes in the DC proteome directly ${ }^{11-14}$ by identifying protein spots on $2 \mathrm{D}$ gels. Due to the limitation of the methods used, however, these studies have so far encompassed expression data of only tens to hundreds of the most abundant proteins or were restricted to partial proteomes of plasma membrane or cytoplasm only. ${ }^{13,15}$

In this study, we have assessed the protein state of human cytokine matured DCs (currently the most widely applied maturation procedure in antitumor immunotherapy ${ }^{6}$ ), by comprehensive quantitative proteomics. Simultaneously, we analyzed the transcriptome of the same batch of cells that was analyzed by proteomics, allowing not only a direct assessment of the correlation between changes in transcription and protein expression but also combination of these complementary data sets to extract biological relevant information. Using integrated pathway analysis we identified 5 pathways that changed significantly in RNA as well as protein expression. Significant enrichment of most established markers of DC maturation within the identified pathways in multiple donors validated that we were able to extract relevant information on DC maturation from as little as one biological sample. We demonstrate that integration of different but related data sets at the pathway level significantly increases the predictive power of multi "omics" analyses.

\section{Materials and Methods}

Cells. For proteomics and microarray analysis dendritic cells were ex vivo generated under serum free conditions from isolated monocytes according to a slightly modified method as described previously. ${ }^{16}$ In short, Peripheral blood mononuclear cells were isolated from the blood of a healthy donor with informed consent by leukaphereses, from which monocytes were extracted using the Elutra system. For differentiation of monocytes to dendritic cells, cells were cultured for 6 days in Cellgro medium (CellGenix, Freiburg Germany) supplemented with IL-4 (300U/ml) and GM-CSF (450U/ml) (both from Strathmann, Hamburg Germany). DC were cultured for
8 days (immature DC) or for 6 days followed by two days in differentiation medium, Cellgro (CellGenix) supplemented with recombinant TNF $\alpha$ (10 ng/mL; CellGenix), IL-1- $\beta$ (5 ng/mL; Immunotools, Friesoythe, Germany), $\mathrm{PGE}_{2}(10 \mu \mathrm{g} / \mathrm{mL}$; Pharmacia \& Upjohn, Puurs, Belgium) and IL-6 (15 ng/mL; Cellgenix) to obtain mature DC. At Day 8 both immature and mature DCs were detached using icecold PBS and taken up in RNeasy (Qiagen) lysis buffer (RLT) for RNA analysis and for proteomics in homogenization buffer (20 mM Hepes (Roche) $\mathrm{pH}$ 7.5, 250 $\mathrm{mM}$ sucrose (Baker, Deventer, The Netherlands) and complete protease inhibitor cocktail (Roche)).

For flow cytometric analysis, monocytes were isolated from a buffy coat obtained from healthy volunteers with informed consent according to institutional guidelines. Monocytes were isolated from peripheral blood mononuclear cells (PBMC) by adherence in RPMI 1640 (Invitrogen life Technologies) containing $2 \%$ Human Serum, as described previously. ${ }^{17}$ Adherent monocytes were subsequently differentiated in the presencese of $450 \mathrm{U} / \mathrm{ml} \mathrm{GM-CSF}$ and $300 \mathrm{U} / \mathrm{mL}$ IL-4 (both from Strahtman, Hamburg, Germany) that was added to either RPMI 1640 containing 10\% Fetal Calf Serum (FCS), $100 \mathrm{U} / \mathrm{mL}$ antibioticantimycotic (Invitrogen), $10 \mathrm{mM}$ Ultraglutamine or to Cellgro medium supplemented with $100 \mathrm{U} / \mathrm{mL}$ antibiotic-antimycotic. After 6 days cells were matured with either cytokine cocktail (see above), a combination of R848 $(4 \mu \mathrm{g} / \mathrm{mL}$ ) (Alexis biochemicals) with Poly I:C (20 $\mu \mathrm{g} / \mathrm{mL}$, Sigma), or a combination of Dexamethasone (100 nM, Sigma) and IL-10 (100 U/mL, Dynax), for $48 \mathrm{~h}$.

Flow Cytometric Analysis. At day 8 cells were detached using cold PBS, fixed in $4 \%$ Paraformaldehyde, permeabilized using $0.1 \%$ Saponin in PBS, $0.5 \%$ BSA and immunostained with antibodies against CCR7 (Clone 150503, R\&D Systems), ICAM-1 (clone rek-1), CXCR4 (clone 2B11, BD Biosciences), CD36 (clone TR9, Biolegend), CD40 (clone MAB89, Beckman Coulter), JAM-1 (clone BV16, a kind gift from Dr. Dejana, Milan, Italy), CD274 (clone MIH1, BD Biosciences), CD31 (clone WM59, BD Biosciences). Cells were analyzed on a FACS Callibur (Beckton Dickinson) and obtained flow cytometric data were analyzed with WinMDI Software.

RNA Analysis. RNA was extracted with an RNeasy kit (Qiagen). Sample preprocessing and biotin labeling were performed using the Affymetrix GeneChip HT One-Cycle Target Labeling and Controls Kit (Affymetrix) according to the manufacturer's protocols. Labeled samples were then hybridized on Affymetrix GeneChip HG U133 Plus 2.0 Arrays, and scanned according to the manufacturer's instructions on a GeneChip Scanner 3000 (Affymetrix).

Transcriptome Data Analysis. CEL files were preprocessed and normalized with the Robust Multiarray Average (RMA) ${ }^{18}$ procedure, in order to obtain absolute-scale expression levels for all genes, using custom chip definition files (CDFs) to update the annotation of the GeneChips, removing duplicate probes and mapping all probes to single Entrez Gene IDs, as previously described by Dai et al. ${ }^{19}$ The computation was performed with the RMAExpress program, version 1.0 beta 10 (http://rmaexpress.bmbolstad.com), on the Linux operating system. Microarray data were filtered to exclude probes whose intensity was less or equal to the background in at least $30 \%$ of the arrays. The operation was performed with the aid of oneChannelGUI, a program part of the Bioconductor suite (http://www.bioconductor.org).

Sample Preparation for Proteomics. $10^{8}$ immature or mature DC were homogenized by 3 cycles of freeze/thawing 
in $500 \mu \mathrm{L}$ homogenization buffer (above) and subsequently fractioned into a soluble and insoluble fraction by centrifugation for 10'at 14000 in an Eppendorp Centrifuge. Samples from $5 \times 10^{5}$ dendritic cells were complemented with $1 / 5$ th volume $5 \times$ Leamli sample buffer containing $\beta$-mercaptoethanol (Sigma) and loaded onto an $4-20 \%$ gradient TRIS/Bis Ready Gel(Biorad). After electrophoreses, protein gels were stained using the Novex colloidal blue staing kit (Invitrogen) and cut into 10 slices per cellular fraction. Gel slices were treated with dithiothreitol (DTT) and iodoacetamide and digested by trypsin as described before. ${ }^{20}$ Digested samples were acidified to a final concentration of $0.5 \%$ HAc and purified by STAGE tips. ${ }^{21}$

Liquid Chromatography Tandem Mass Spectrometry. Peptide sequencing experiments were performed using a nano HPLC Agilent 1100 flow system connected online to a 7-T linear ion trap ion cyclotron resonance Fourier transform (LTQ-FT) mass spectrometer (Thermo Fisher, Bremen, Germany). Digested samples were measured up to three times to increase the number of low abundant protein identifications. Peptides were separated on a $15 \mathrm{~cm} 100 \mu \mathrm{m}$ ID PicoTip columns (New Objective, Woburn, MA) packed with $3 \mu$ m Reprosil C18 beads (Dr. Maisch GmbH, Ammerbuch, Germany) using a $90 \mathrm{~min}$ gradient from $12 \%$ buffer B to $40 \%$ buffer B (buffer B contains $80 \%$ acetonitrile in $0.5 \%$ acetic acid) with a flow-rate of 300 $\mathrm{nL} / \mathrm{min}$. Peptides eluting from the column tip were electrosprayed directly into the mass spectrometer with a spray voltage of $2.0-2.2 \mathrm{kV}$. Data acquisition with the LTQ-FT instrument was performed in a data-dependent mode to automatically switch between MS and MS2. Full-scan MS spectra of intact peptides $(\mathrm{m} / z 350-1500)$ with an automated gain control accumulation target value of 1.000 .0000 ions were acquired in the Fourier transform ion cyclotron resonance cell with a resolution of 50.000. The four most abundant ions were sequentially isolated and fragmented in the linear ion trap by applying collisionally induced dissociation using an accumulation target value of 10.000 , a capillary temperature of $100{ }^{\circ} \mathrm{C}$, and a normalized collision energy of $27 \%$. A dynamic exclusion of ions previously sequenced within $180 \mathrm{~s}$ was applied. All unassigned charge states and singly charges ions were excluded from sequencing. A minimum of 500 counts was required for MS2 selection.

Peptide Identification by MASCOT Database Searches. RAW spectrum files were converted into a Mascot generic peaklist by DTA supercharger v1.18 (www.msquant.sourceforge.net). Proteins were identified by searching peak lists containing fragmentation spectra with Mascot version 2.1 (Matrix Science) against a local version of the human International Protein Index (IPI) database version 3.25 (ftp://ftp.ebi.ac.uk/pub/databases/ IPI/) that contains 67250 protein entries. A reversed protein sequence database was searched to determine the falsediscovery rate of protein identifications. The reversed protein database was created by the decoy.pl script provided by Matrix Science (http://www.matrixscience.com/help/decoy_help. html). Mascot search parameters for protein identification specified an initial mass tolerance of $30 \mathrm{ppm}$ for the parental peptide and $0.5 \mathrm{Da}$ for fragmentation spectra and a trypsin specificity allowing up to 3 miscleaved sites. Carbamidomethylation of cysteines was specified as a fixed modification, and oxidation of methionine, deamidation of glutamine or asparagine were set as variable modifications. Modification sites were determined by the PTM scoring algorithm from the MSQuant v 1.31 software package (www.msquant.sourceforge. net). Internal mass calibration of measured ions was also performed with MSQuant, that resulted in a an average peptide recalibrated mass accuracy of $3.23 \mathrm{ppm}$ with a final absolute recalibrated mass tolerance for the parental peptide of $10 \mathrm{ppm}$. We accepted only multiple charged peptides with precursor masses larger than 350 and a minimal Mascot peptide score of 22. Reverse database searches revealed no false-positive identifications for proteins identified by 3 or more nonredundant peptides by these criteria. More stringent criteria were required to identify proteins sequenced by one or two peptides with high confidence. A false-discovery rate of $0.26 \%$ was determined for proteins identified by 2 unique unmodified peptides, and a false-discovery rate of $0.75 \%$ was obtained for proteins identified by 1 unmodified peptide with a peptide cut off score of 40, and a Mascot peptide delta score $>10$. To unambiguously map a peptide sequence on a protein sequence, peptides were remapped to proteins of IPI database version 3.25 using the program Protein Coverage Summarizer (http:// ncrr.pnl.gov/software/). Peptide and protein lists are provided as Supporting Information. Conversion of IDs (IPI to Entrez IDs) was performed with the biomaRt Bioconcuctor package.

emPAI Analysis to Determine Protein Abundance Profiles. Human DC differentiated from monocytes in vitro do not divide and therefore metabolic labeling methods for quantitative mass spectrometry cannot be used. As an alternative, we used a peptide counting method first described by Mann and colleagues (exponentially modified protein abundancy index EmPAI ${ }^{22,23}$ that does not rely on labeled samples for quantitative analysis. To determine protein abundance values in our samples, validated mass spectrometric data was quantified by the computation of emPAI values for all proteins. EmPAI values were calculated according to the formula:

$\mathrm{emPAI}=10^{\mathrm{PAI}}-1$, with $\mathrm{PAI}=n_{\text {observed peptides }} / n_{\text {observable peptides }}$

The number of "observable" peptides per protein was calculated from the output of the program Protein Digestion Simulator (http://ncrr.pnl.gov/software/), which computes peptide masses and hydrophobicities of simulated digests of protein databases. EmPAI values were calculated for proteins detected in all individual gel slices measured in triplicate. Normalization between runs was performed on the mean emPAI value of the 11 most abundant proteins detected in DCs, resulting in an overall Coefficient of Variation of 0.292 . This normalization method performs the best (lowest CV) in the methods we tested, that were based on either total, median or mean intensity of all proteins (data not shown). For those proteins that were only detected in either immature or mature DCs, a minimal EmPAI value was calculated for missing data based on the assumption that 1 peptide was detected, allowing us to calculate a ratio corresponding to the minimal effect (thus underestimating the real effect).

Calculation of Overlap between Proteome and Transcriptome. Venn diagrams, to display overlaps among expression and protein data, were drawn using the limma package. Comparisons between proteomics and transcriptomics data were performed either using the full transcriptomic and proteomics data, or only with genes (or proteins) with a fold change of 2 or greater. Likewise, an identical approach was used with the results of pathway analysis (see below), including either all entities (genes and proteins) or only the ones with a fold change of 2 or greater. All the computations were performed using the $\mathrm{R}$ programming language. 
Correlation Analysis. Pearson's correlation was calculated between the transcriptomic and proteomics data using the $\mathrm{R}$ programming language (corr and corr.test functions). The calculation of the correlation coefficient $r$ was done using the following formula:

$$
r=\frac{\sum X Y-\frac{\sum X \sum Y}{N}}{\sqrt{\left(\sum X^{2}-\frac{\left(\sum X\right)^{2}}{N}\right)\left(\sum Y^{2}-\frac{\left(\sum Y^{2}\right.}{N}\right)}}
$$

Selection of the Pathway Set. To identify biologically meaningful changes in our data sets, we built a pathway set, result of a curation by experts of the DC-THERA European Network of Excellence. Out of the 1038 human pathways available, a selection process was performed in order to identify the most relevant pathways from an immunological point of view. The result was a set of 29 pathways collected from the KEGG and Reactome public databases, which satisfied those criteria.

Pathway Analysis. Eu.Gene ${ }^{24}$ was used to perform the analysis on the selected pathway set using the Fisher's Exact Test (FET) ${ }^{25}$ as the analysis algorithm. As an alternative also the Functional Annotation web tool David (http://david.abcc. ncifcrf.gov/) was used to calculated EASE scores (a modified one-tailed fisher exact test ${ }^{26,27}$ (Table S5, Supporting Information).

Fisher's Exact Test. Treated and untreated sample data were separated, and the expression level of each gene in each treated sample was divided by the expression level of the same gene in the corresponding paired untreated sample, and the resulting ratio was $\log _{2}$ transformed, obtaining a list of $\log _{2}$ ratios for each gene in each treated sample. The transformed expression data were then used for Fisher's Exact Test. Ratios originating from proteomics data (see above) were used directly for the analysis without any transformation. The upper and lower cutoffs for FET were determined from the $2 \sigma$ interval of the binomial distribution of expression values. ${ }^{28}$ The FET $p$-value for each pathway was calculated as

$$
p=1-\sum_{i=0}^{a} \frac{\underset{i}{(S)}(\underset{p-i}{F})}{(\underset{P}{N})}
$$

where $S$ is the number of genes that the calculated pass the cut-offs, $F$ is the number of genes that do not pass the cutoffs, $N$ is the total number of genes in the array, $P$ is the number of genes that belong to the pathway, and NP is the number of genes that do not belong to it. The algorithm was then run using the hypergeometric distribution without approximation. Each pathway was associated to a signed $p$-value and a matrix of signed $p$-values for all samples was obtained for each data set. To provide a correction for multiple testing, $p$-values were adjusted following the procedure by Benjamini et al. ${ }^{29}$

Data Deposition. RNA microarraydata is deposited at ArrayExpress (http://www.ebi.ac.uk/microarray-as/ae/; accession number E-MEXP-2290) and proteomics data is deposited at Human Proteinpedia (http://humanproteinpedia.org; ${ }^{30}$ accession numbers *HuPA_00661* (for immature DC) and *HuPA_00662* (for mature DC).

\section{Results}

From immature DCs and cytokine cocktail (Il-1b, TNF-a, IL6, PGE2) matured human monocyte derived DCs we obtained expression information for more than 16000 genes at the RNA level and for 2695 genes at the protein level (Tables S2-S4, Supporting Information). Because our main focus is to improve the extraction of reproducible information from immature and mature DCs from a single donor by integrating different types of information, we did not retrieve data from biological replicates and expression ratios were thus left without calculation of significance at the single gene or protein level. Calculations however were performed using stringent statistical criteria to ensure highest data quality (see Methods) and most important biological information was ultimately verified on DCs from multiple individuals (below) to ensure widespread significance.

At first glance, changes in expression in both data sets were consistent with known maturation markers and with the notion that during maturation DCs switch from a sensory state toward a migratory and finally $\mathrm{T}$ cell stimulatory state: Expression of migration factor CCR7 and T cell-stimulatory factors CD40 and ICAM-1 (CD54) were up-regulated while expression of endocytic receptors that included DC-SIGN (CD209) and CD36 was down-regulated (Table S2, Supporting Information). Overall, the majority of proteins and mRNAs decreased in expression after the induction of maturation. A specific subset however, containing among others proteins involved in T cell stimulation, was up-regulated suggesting "end point" specialization of the DCs by aborting the expression of proteins no longer needed upon maturation and reorienting synthesis on those proteins needed to perform the specific function of mature DCs (i.e., migration and antigen presentation).

Our proteomics data identified peptides and proteins that were mapped to the human International Protein Index (IPI) protein database whereas genes of our transcriptomics data were mapped to Affymetrix probe identifiers (IDs) by a proprietary identification system that maps to well-known international standards for transcript identification. To directly compare transcription data with proteomics data, conversion to a common ID was required for which we chose the widely used Entrez gene ID. ${ }^{19}$ We obtained 16383 Entrez IDs for the microarray data and 2638 Entrez IDs for the proteomics data, of which 2480 were shared between RNA and protein data sets and could thus be directly compared (Tables 1 and S4, Supporting Information). To quantify the proteomics data, we used the recently developed label free exponentially modified Protein Abundance Index (emPAI) method. ${ }^{22,23}$

To assess covariation we calculated the Pearson correlation between the gene and protein expression ratio's for mature versus immature DCs of the 2480 IDs corepresented in both data sets and found a relatively low correlation coefficient (0.38). This is not unexpected as mRNA expression in time precedes protein expression and in addition protein and corresponding mRNA molecules may have a very different turnover. Nonetheless, we expected that for a large number of proteins important for DC function $48 \mathrm{~h}$ after the induction of maturation, protein expression is maintained at high levels by continuous mRNA transcription. Indeed, we found 283 genes that are coregulated at the mRNA and protein level to be differentially expressed into the same direction (up or down; Figure 1; Table S4, Supporting Information). Excluded in Figure $1 \mathrm{~B}$ are 44 proteins whose expression was oppositely regulated with respect to gene expression (17 up and 27 down in protein expression (Table S4, Supporting Information)). 
A Total
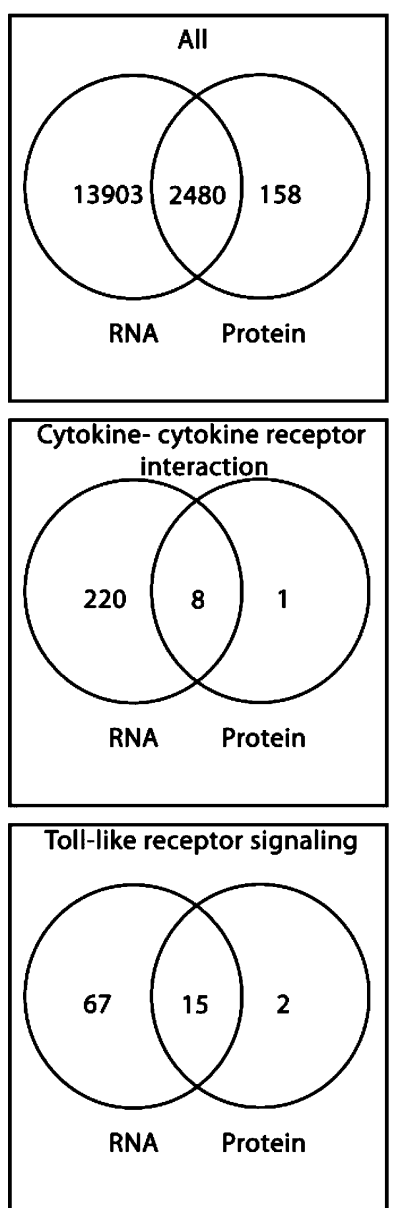
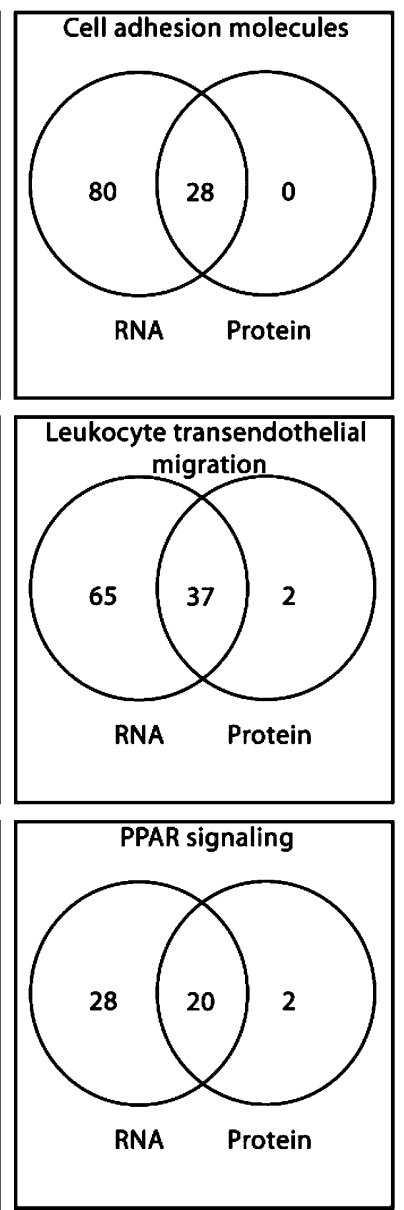

B
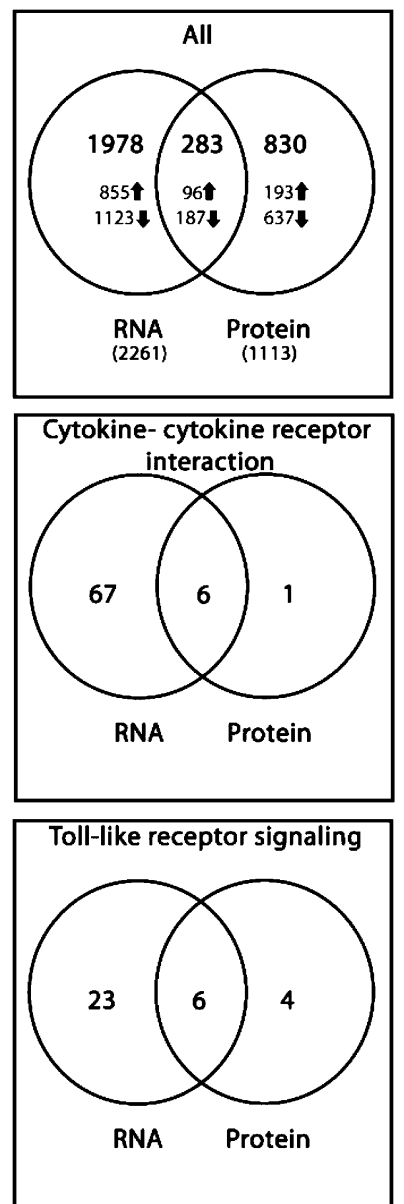

$>2$ fold changed
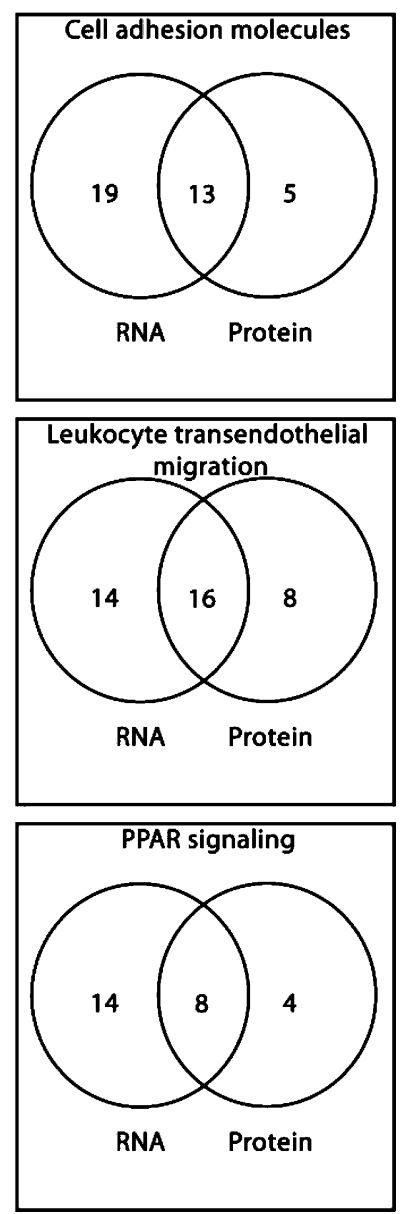

Figure 1. Overlap between RNA and protein expression during DC maturation. Shown are Venn diagrams for all proteins and RNA data sets and for the components of 5 dominant pathways of DC maturation (Table 1). (A) RNA and protein counts for the total of expressed genes and proteins. (B) Subset of differentially $(>2)$ expressed genes/proteins.

Table 1. Dominant Pathways in DC Maturation ${ }^{a}$

\begin{tabular}{|c|c|c|c|c|c|c|c|}
\hline pathway name & FET RNA & $\begin{array}{c}\text { changed } \\
\text { components RNA }\end{array}$ & FET protein & $\begin{array}{l}\text { changed components } \\
\text { protein }\end{array}$ & $\begin{array}{c}\text { shared } \\
\text { components }\end{array}$ & $\begin{array}{l}\text { Pearson r } \\
\text { correlation }\end{array}$ & $\begin{array}{l}\mathrm{p} \text {-value } \\
\text { correlation }\end{array}$ \\
\hline Leukocyte transendothelial migration & $2.1 \times 10^{-3}$ & 30 & $5.02 \times 10^{-3}$ & 24 & 37 & $6.4 \times 10^{-1}$ & $2.0 \times 10^{-5}$ \\
\hline Cytokine-cytokine receptor interaction & $1.6 \times 10^{-7}$ & 73 & $5.92 \times 10^{-3}$ & 7 & 8 & $9.7 \times 10^{-1}$ & $4.4 \times 10^{-5}$ \\
\hline Cell adhesion molecules (CAMs) & $1.9 \times 10^{-3}$ & 32 & $5.76 \times 10^{-3}$ & 18 & 28 & $6.8 \times 10^{-1}$ & $6.2 \times 10^{-5}$ \\
\hline $\mathrm{T}$ cell receptor signaling pathway & $2.4 \times 10^{-4}$ & 29 & & & 18 & $7.7 \times 10^{-1}$ & $2.0 \times 10^{-4}$ \\
\hline Natural killer cell mediated cytotoxicity & $5.0 \times 10^{-2}$ & 27 & & & 23 & $6.9 \times 10^{-1}$ & $2.8 \times 10^{-4}$ \\
\hline Apoptosis & $5.6 \times 10^{-5}$ & 28 & & & 25 & $5.8 \times 10^{-1}$ & $2.3 \times 10^{-3}$ \\
\hline B cell receptor signaling pathway & $1.3 \times 10^{-4}$ & 22 & & & 17 & $6.7 \times 10^{-1}$ & $3.2 \times 10^{-3}$ \\
\hline Focal adhesion & $6.7 \times 10^{-2}$ & 39 & & & 40 & $4.1 \times 10^{-1}$ & $8.9 \times 10^{-3}$ \\
\hline Jak-STAT signaling pathway & $1.1 \times 10^{-4}$ & 42 & & & 13 & $5.6 \times 10^{-1}$ & $4.5 \times 10^{-2}$ \\
\hline
\end{tabular}

${ }^{a}$ All DC relevant pathways with a significant correlation between RNA and protein expression changes are shown $(p<0.05)$. Those pathways that were significantly enriched in changes at both the RNA and protein level (RNA $p<0.01$ and protein $p<0.1$ ) are listed in bold.

Obviously, in the absence of biological replicates single gene analysis will likely not have much predictive power. Therefore, we next focused at the coherency between mRNA and protein changes at the level of biological pathways to obtain a more general comparison of mRNA and protein data with respect to processes affected during DC maturation. We determined the correlation between differentially expressed proteins and genes for the grouped components of preassembled publicly available pathways. We anticipated that for elements of pathways important for DC function $48 \mathrm{~h}$ after maturation, the correlation between mRNA and protein expression changes might be higher than the overall correlation (elements not grouped in pathways), since these pathways likely contain many differentially expressed elements. Vice versa, the correlation of less important pathways is expected to be low because these will only contain elements that are not changed at all (clustering 
Table 2. Differentially Expressed Proteins from Dominant Pathways in DC Maturation ${ }^{a}$

\begin{tabular}{|c|c|c|}
\hline pathway & 2-fold down & $>2$-fold up \\
\hline Cell Adhesion molecules & $\begin{array}{l}\text { Golgi apparatus protein } 1 \text { (p) -- PECAM } \\
\text { (CD31) (b)-Sialoadhesin (SIGLEC1, } \\
\text { CD169)(b)--Integrin beta 1 isoform 1B } \\
\text { (CD29)(p)--CD276 antigen isoform a } \\
\text { (B7-H3)(b)--Claudin } 15 \text { (p)--ALCAM } \\
\text { (p)--CD4 antigen precursor (b)--Integrin, } \\
\text { beta } 2 \text { (CD18)(b)--Sialophorin } \\
\text { (CD43) (b)--Integrin alpha M (CD11b) } \\
\text { (b)--Integrin alpha L--(CD11a) (b)--HLA II, } \\
\text { DRa (p)--CD273 (PDL2) (p) }\end{array}$ & $\begin{array}{l}\text { ICAM-1 (b)--CD274 (PDL1)(b)--JAM1 } \\
\text { (b)--CD40 (b) }\end{array}$ \\
\hline Toll like receptor signaling & $\begin{array}{l}\text { Tollip (b)--MAPK14 (p38)(p)--TICAM2 (p)-- } \\
\text { STAT1 (b)--IKB (p) }\end{array}$ & $\begin{array}{l}\text { PI-3-kinase, regulatory subunit } 1 \text { (p)--RELA } \\
\text { (b)--CD40 (b)--NFKB1(b)--NFKB2 (b) }\end{array}$ \\
\hline PPAR signaling & $\begin{array}{l}\text { Acetyl-Coenzyme A acyltransferase } 1 \text { isoform } \\
\text { a (p)--Acyl-CoA synthetase long-chain } \\
\text { family member } 5 \text { (b) --APO A-I (p)--CD36 } \\
\text { (b) --FABP4 (b) --Malic Enzyme } \\
\text { (b)--Acyl-Coenzyme A dehydrogenase, C-4 } \\
\text { to C-12 (b)--Acyl-Coenzyme A oxidase 1, } \\
\text { palmitoyl isoform b (p)--Mitochondrial } \\
\text { phosphoenolpyruvate carboxykinase (b) }\end{array}$ & $\begin{array}{l}\text { Glycerol kinase (b)--Diazepam binding } \\
\text { inhibitor (p)--Acyl-CoA synthetase } \\
\text { long-chain family member } 4 \text { (b) }\end{array}$ \\
\hline Leukocyte transendothelial migration & $\begin{array}{l}\text { PECAM (CD31) (b)--Integrin beta } 1 \text { isoform } \\
\text { 1B (CD29) (p)--ROCK1 (p)--RAP1A } \\
\text { (b)--Neutrophil cytosolic factor } 2 \text { (p67phox) } \\
\text { (p)--VASP (p)--MAPK14 (p38)(p)--Claudin } \\
\text { 15 (p)--Vav 1 (b)--Cytochrome } b \text {-245 beta } \\
\text { polypeptide (NOX2) (b)--Integrin, beta } 2 \\
\text { (CD18) (b)--Integrin alpha M } \\
\text { (CD11b)(b)--RAP1B (p)--Catenin, delta } 1 \\
\text { isoform 1B (b)--G protein alpha } \\
\text { (p)--Myosin, light chain } 6 \text { (p)--Neutrophil } \\
\text { cytosolic factor } 4 \text { (p40 phox) (b)--Integrin } \\
\text { alpha L isoform a (CD11a) } \\
\text { (b)--Phospholipase C, gamma } 2 \text { (b) }\end{array}$ & $\begin{array}{l}\text { PI-3-kinase, regulatory subunit } 1 \text { (p)--CXCR4 } \\
\text { (b)--PTK2B protein tyrosine kinase } 2 \text { beta } \\
\text { (b)--JAM1 (b)--ICAM-1 (b) }\end{array}$ \\
\hline Cytokine-cytokine receptor interaction & $\begin{array}{l}\text { Colony stimulating factor } 1 \text { receptor } \\
\text { (b)--CCL18 (DC-CK1) (b) }\end{array}$ & $\begin{array}{l}\text { CXCR4 (b)--CCL17 (b)--CD40 (b)--colony } \\
\text { stimulating factor } 2 \text { receptor alpha chain } \\
\text { (p)--CCR7 (b) }\end{array}$ \\
\hline
\end{tabular}

\footnotetext{
${ }^{a}$ Included in the table are pathway elements differentially expressed both at the RNA and protein level (indicated by a (b) after the protein name) or differentially expressed at the protein level only (indicated by a (p)).
}

together in a correlation plot) or are uniformly down-regulated as a result of "end point specialisation". We thus calculated Pearson correlation coefficients for all biological pathways annotated in the Kegg database and for those selected as "relevant" for dendritic cell function by the DC-ATLAS working group in the DC-THERA consortium (http://www.dc-thera.org/; Table S5, Supporting Information). As expected, the Pearson correlation coefficient for the total of elements belonging to the grouped biological pathways relevant for DCs was indeed improved (0.55) compared to the complete set of pathways (0.44) although this difference was not significant. However, for some pathways the correlation was extremely high $(0.97$; see Table 1). Such high correlations were not likely found by chance as we determined by calculating the correlation of random, similarly sized groups of elements from our data set. (Tables 1 and S5 and Figure S1, Supporting Information). These findings indicate that after $48 \mathrm{~h}$ of maturation, DC relevant pathways are more regulated by transcriptional control than pathways less important to DC maturation at this time point.

Next, for all DC relevant pathways with a significant correlation $(p<0.05)$, we calculated the relative amount of differentially expressed genes or proteins by pathway enrichment analysis, using either the Functional Annotation web tool David (http://david.abcc.ncifcrf.gov/) or the novel Eu.Gene software. ${ }^{24}$ The latter tool also provides a statistically valid $p$-value with a positive or negative value to indicate the direction of change (Note: This sign preceding the $p$-value is not intended to imply anything on the statistical value, that is indicated by the $p$-value alone). Fisher exact tests (FET) performed with both programmes essentially yielded similar results (Table 1 and Table S5, Supporting Information), although results with Eu.Gene were statistically more significant because it allows restriction of multiple-testing correction to the number of actual pathways tested. Exploiting these methods we were able to select 5 major pathways (Table 1), sustained by active transcription and affected significantly at both protein and RNA level, representing the dominant cellular processes that determine function and activity of DCs $48 \mathrm{~h}$ after providing maturation stimuli.

The 5 pathways we identified represented: "Toll like receptor signalling (TRS)", "Cytokine-cytokine receptor interaction (CCI)", "Cell adhesion molecules (CAM)", "Leukocyte Transendothelial migration (LTM)", and "PPAR signalling (PPAR)". The first two pathways contained relatively more up-regulated genes and proteins, whereas the latter three contained more down-regulated components (Tables 1, 2, and S6, Supporting Information). That this combination of protein and RNA data sets at the pathway level very efficiently guided us to the most 

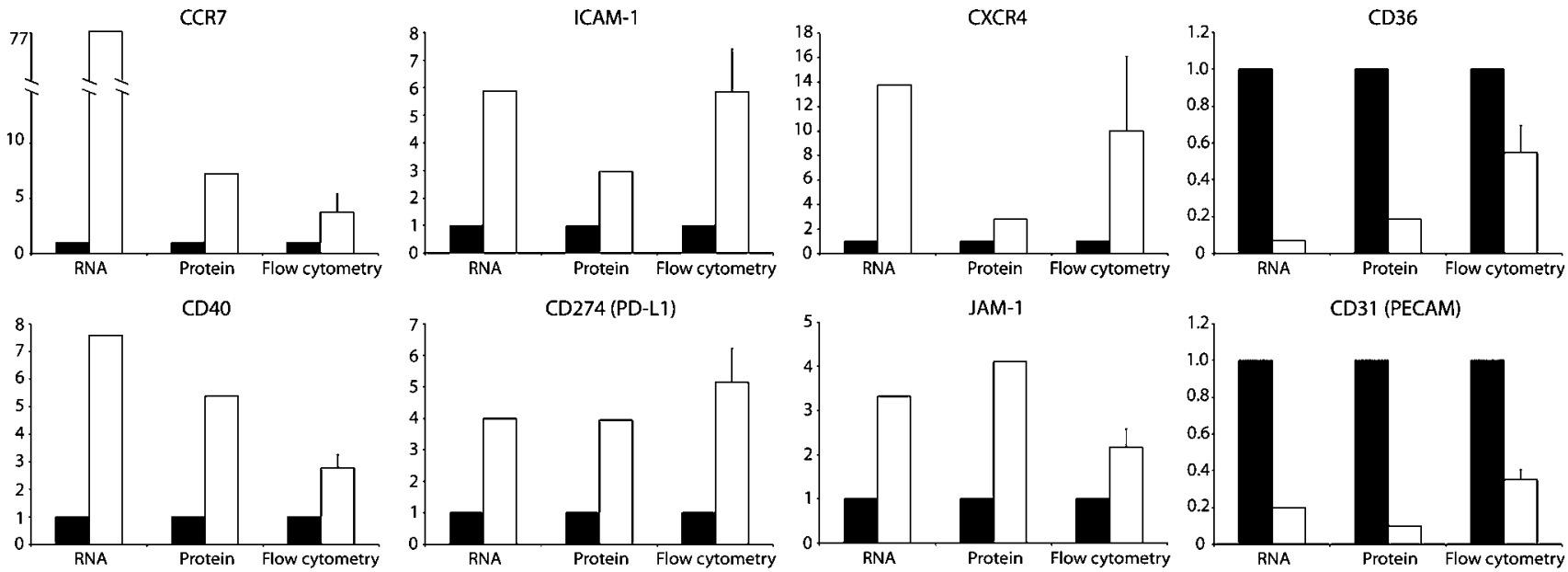

Figure 2. RNA and protein marker expression by transcriptomics/proteomics from a single donor compared to expression by flow cytometric analysis from DCs of multiple donors: Expression ratio's of the microarray (RNA) and proteomics (protein) data was compared to flow cytometric analysis of fixed and permeablized DCs from 4 independent donors (fold change relative to immature DC \pm s.e.m.). Day $8 \mathrm{immature} \mathrm{DC}$ are respresented by the filled bars and day 8 cytokine cocktail matured DC are indicated by the empty bars. Shown are 5 well-known maturation markers (CCR7, ICAM-1, CD40, CD36 and CXCR4) and 3 potential novel markers (JAM-1, PD-L1 and CD31).

important processes for DC maturation is clearly illustrated by the presence of most established maturation markers within these 5 pathways (e.g., CD83, CD86, HLA I and II, CCR7, ICAM-1 and others; Table 2, Table S6, Supporting Information and ref 31). To demonstrate this in a more quantitative manner, we used a "maturation marker set" that we extracted from a well cited review on DC characteristics in immunotherapy composed of 20 markers (Table S7, Supporting Information and ref 31). We tested whether this marker set was indeed enriched in the 5 selected pathways relative to the whole data set and found the marker set to be 30 fold enriched ( $p<0.001$ ), a value that could never be obtained using either RNA (16-fold enrichment) or protein data (7 fold enrichment) alone (Table S8, Supporting Information). By combining RNA and protein data sets we are thus able to select more precisely pathways containing DC maturation markers. The content of these pathways now provides a novel rich data source for extracting potentially important new marker proteins to monitor DC quality for immunotherapy.

So far these analyses allowed selection of the most important pathways, but we cannot determine whether the individual differentially expressed elements within these pathways are the same for RNA and protein. The correlation analysis only takes into account those elements present in both data sets, whereas the pathway enrichment analysis only takes into account the number of differentially expressed elements but not their origin. To, reveal to what extent proteomics and microarray data only support or also complement each other, we thus analyzed the selected pathways in depth at the individual gene and protein level. While most pathway elements identified at the protein level were also supported by gene expression regardless of the pathway they belonged to, this was as can be expected due to the much lower number of proteins detected, often not the case for elements identified at the gene expression level (Figure 1). We observed that, for the 5 pathways associated with DC maturation, changes were also to a variable extend restricted to protein expression. Only for the CCI pathway almost no differential expression was detected uniquely at the protein level. For the other pathways on the other hand, several unique protein expression changes were found. In these cases, the significant pathway enrichment calculated with Eu.Gene (the FET $p$-value) thus relied also to a large extend on unique differential protein expression changes occurring in both gene and protein expression (Figure 1 and Table 1). Therefore, by combining RNA with protein expression data we gained information on additional pathway elements. To appreciate overlapping and uniquely expressed elements also in the pathway context we, as an example, visualized differential RNA and protein expression within the TRS pathway (Figure S2, Supporting Information).

So far, at the pathway level we compared RNA and protein expression on cells from one single human healthy donor. At the single protein level our integrated "omics" analysis however does not compensate for variations in expression between donors. To validate our most important findings, we next performed flow cytometric analyses and measured expression levels of specific proteins on DCs derived from several healthy individuals (Figure 2). We selected proteins from the 5 pathways identified that showed differential expression both at the RNA and the protein level after DC maturation and may therefore be regarded as potential novel maturation markers. In order to detect total protein levels and allow comparison to the whole cell with protein expression data obtained by proteomics, we used fixed and permeabilized cells. The flow cytometetric analysis was in complete agreement with proteomics data of Jam-1, CD274 (PD-L1) and CD31 (PECAM-1). As a positive control we also selected one well-defined marker for every selected pathway important for DC maturation (Figure 2) and again flow cytometry confirmed differential protein expression levels as determined by proteomics of CCR7, CD40, CXCR4, ICAM-1 and CD36.

Finally, to determine whether these potential novel marker proteins are indeed associated with DC maturation and activation, we compared alterations in expression levels resulting from treatment of DCs with the clinically used cytokine cocktail, but also with different maturation stimuli; a potent immunogenic Toll Like Receptor (TLR) stimulus (Poly I:C combined with R848) and a potent tolerogenic stimulus (Il-10 combined with dexamethasone). As expected TLR stimulation changed expression of conventional maturation markers ICAM- 

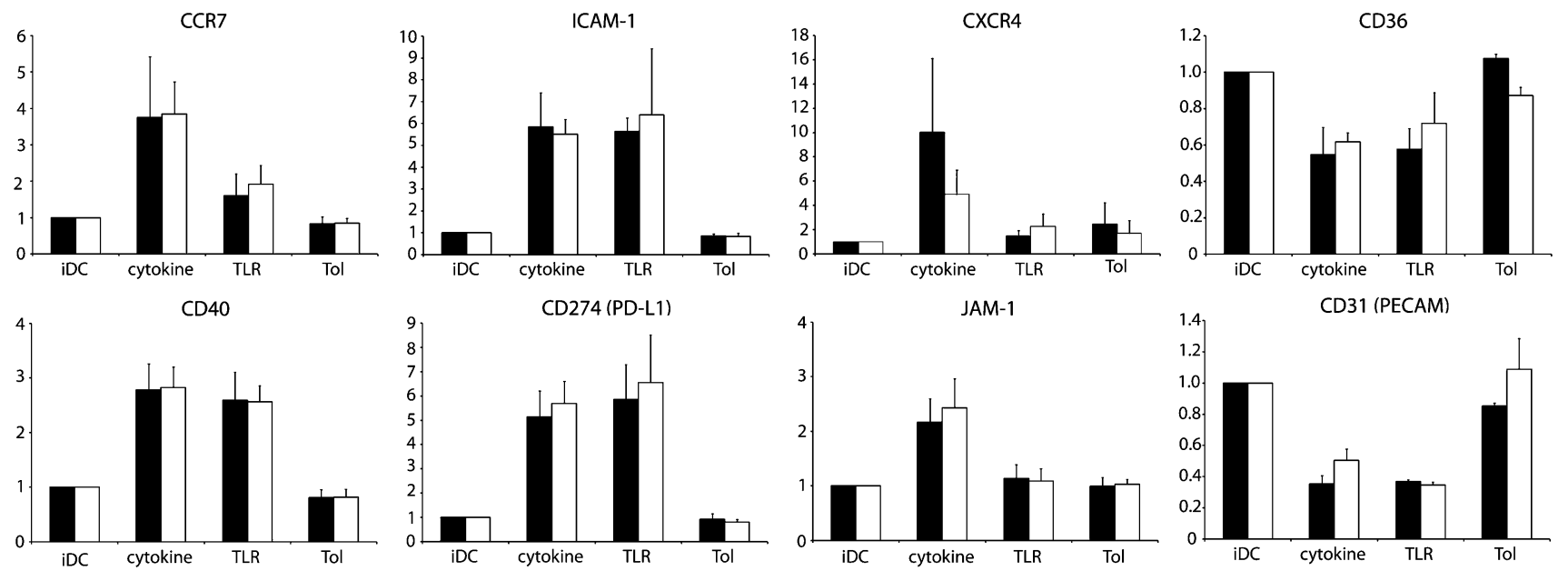

Figure 3. Marker expression in response various DC stimulation cocktails: Marker expression relative to immature DC (iDC) was monitored by flow cytometric analysis of fixed and permeablized DCs from 4 independent donors (fold change relative to immature $D C \pm s . e . m$.)) after stimulation with the cytokine cocktail, the immunogenic combination of R848 and Poly I:C (TLR), or a combination of the tolerogenic compounds IL-10 and dexamethasone (TOL). Cells cultured in serum free Celgro medium are indicated by the filled bars and cells grow in RPMI $+10 \%$ FCS are represented by the empty bars.

1, CD40 and CD36 similar to cytokine-induced maturation (Figure 3). Tolerogenic stimulation of DCs had no or even opposite effects on these markers. CCXR4 and CCR7 expression levels increased less upon TLR stimulation like reported before. $^{32}$ The potential novel maturation markers CD274 and CD31 behaved similarly when comparing the TLR ligand stimulated DC with the cytokine cocktail matured DCs. Jam-1 on the other hand responded similar to CCR7 and CXCR4 and was hardly affected when DCs were exposed to the potent immunogenic TLR stimulus. This indicates that the increased expression of the latter protein seems more specific for cytokine matured DCs. Additional experimental work beyond the scope of this paper is now required to precisely link the novel markers to immunity or tolerance.

At the same time, we also tested whether the observed effect was specific for cells grown in serum free medium, the "clinical grade" culture condition for the cells analyzed by proteomics and transcriptomics, or also applied to more commonly used culture conditions, in medium supplemented with fetal calf serum. We found no differences between culture conditions tested (Figure 3).

Taken together, the flow cytometric analysis fully supports pathway based integrated analysis of changes in the DC proteome and transcriptome, demonstrating that the identified pathways may serve as valuable resources for the identification of novel marker proteins to guide immunotherapy.

\section{Discussion}

To the best of our knowledge we present for the first time a comprehensive genome-wide expression analysis of human DC maturation on gene and protein level. Previous studies addressing quantitative protein level changes induced by human DC maturation were restricted to those proteins that could be extracted from 2-D SDS gels or that were derived from either the plasma membrane or from cytosol only. ${ }^{11-15}$ These methods yielded quantitative information on a much smaller number than this study. By exploiting the label free quantification method emPAI ${ }^{22,23}$ we are now able to obtain quantitative information of more than 2600 proteins which we supplemented with mRNA expression data of over 16000 genes.
To extract the most relevant information for DC maturation from this large amount of protein and mRNA data, we used the concept that genes rarely act alone but rather together drive cellular processes to establish DC function. Coordinated regulation of groups of genes may be equally or even more important than changes in expression of one single gene or protein, especially when comparing intrinsically different data sets. ${ }^{10}$ At the mRNA level, data sets of comparable biological samples gathered in different laboratories, using various array platforms or analyzed with different statistical methods, may at a single gene level often seem dissimilar but show similar functionality at the pathway level. ${ }^{10}$ We here demonstrate that this now also holds for the comparison of protein and mRNA expression data.

When all overlapping gene IDs between transcriptome and proteome were taken into account mRNA and protein correlation was rather poor, as also observed in other comparative studies. ${ }^{33}$ These inconsistencies between data sets may be explained by differences in timing or stability of protein with respect to mRNA, repression of mRNA translation, or by effects of posttranscriptional regulation. The low correlation may also be a consequence of the end point specialization of the DC after maturation, resulting in data sets biased for down regulation. We found that correlation between transcription and proteomics data improved considerably by focusing the analysis on elements of preassembled publically available pathways describing DC relevant processes indicating most important pathways are more regulated by transcription. Further selection based on the assumption that most important pathways should contain a significantly higher number of differentially expressed proteins as well as genes, resulted in 5 pathways that contribute most to DC maturation $48 \mathrm{~h}$ after the initiation of maturation.

Four out of the five pathways that matched our selection criteria describe processes that can be easily recognized as hallmarks of maturing DCs. ${ }^{1,34}$ LTM and CAM pathways describe events required for migration from peripheral tissues to draining lymph nodes during which DC associate with cells (e.g., endothelium and other immune cells) and the extracellular matrix. These two pathways contain some overlapping 
proteins, which is not unexpected since they describe closely related processes that are connected in time and space. This also true for the TRS and CCI pathways, that both describe signaling events affected by the cytokine cocktail used to mature the DC and their responses to other cell surface receptors and cytokine secretion respectively, ultimately driving $\mathrm{T}$ cell stimulation and T cell skewing (e.g., Th1/Th2/Th17/Treg).

Our analysis also shows that elements of the PPAR signaling pathway alter significantly upon DC maturation. One of the nuclear receptors central to this pathway, $\operatorname{PPAR} \gamma$, acts as a sensor for fatty acids and controls lipid storage and adipocyte differentiation. ${ }^{35}$ PPAR $\gamma$ was recently shown to be involved in immune regulation, providing a possible link between diet and the immune system. ${ }^{36-39}$ During differentiation of monocytes to DCs in vitro PPAR $\gamma$ expression is induced and expressed $\operatorname{PPAR} \gamma$ can subsequently be activated by serum derived ligands, thereby promoting changes in expression of genes related to both immune response and lipid metabolism. ${ }^{40}$ Our results indicate that cytokine stimulation may in part counteract the effects of PPAR $\gamma$, possibly trough the action of TNF- $\alpha$ that is present in the cytokine cocktail. ${ }^{41}$ Although no protein expression was detected for $\operatorname{PPAR} \gamma$, we found its RNA expression to be down-regulated. In addition, for many genes shown to change in response to $\operatorname{PPAR} \gamma$ activation during DC differentiation $^{40}$ we now observed an opposite effect on gene and/or protein expression as a result of DC maturation, further suggesting maturation leads to reduced PPAR activity. We observed a decrease rather than an increase in proteins involved in adhesion (LFA-1, CD43) lipid uptake and metabolism (CD36, FABP4, ME) and an increase instead of a decrease for metalloproteases (MMP1, MMP10 (RNA only)) and several immune related proteins (CD274, CD39, CCR7). Our results suggest that DC maturation causes significant alterations in lipid uptake, metabolism and distribution. From the many players implicated in the PPAR signaling pathway it is at this point difficult and beyond the scope of this paper to dissect the precise nature of the changes as well as their relation to DC immune function.

The selected pathways were found to be highly enriched for established marker proteins currently used to monitor DC maturation (Supporting Information, www.kegg.com and refs 1, 2 and 31) but also contain many proteins with currently unknown function in DC maturation implicating that these pathways potentially can be used to extract novel prognostic factors for DC function. Inititial flow cytometric analysis of DCs from multiple individuals indeed clearly confirmed differential expression of the three potential novel marker proteins PD-L1 (CD274), CD31 and JAM1. Together with the significant enrichment of well-known markers in the selected pathways this further demonstrates that our results obtained with cells from a single donor are of general use.

All three potential marker proteins could have important implications for DC migration and DC induced T cell activation. First our data indicate that both the cytokine cocktail and TLR ligands but not tolerogenic stimuli dramatically upregulates PDL1 expression in DCs. The programmed death ligand-1 (PDL1 or CD274) has been reported as constitutively expressed on a variety of immune cells including human and mouse DCs, and in some cells the expression level can be increased in response to IFN- $\alpha$ and IFN- $\gamma .{ }^{42-44}$ Neither the response to our cytokine cocktail nor the response TLR stimuli, however, has yet been documented for human monocyte derived DC. PDL1 on DCs can bind to either PD-1 or B7.1 on T cells and binding of PD-L1 to both receptors has been shown to inhibit $\mathrm{T}$ cell cytokine production, although $\mathrm{T}$ cells stimulatory effects have also been reported. ${ }^{45,46}$ The expression on DC could thus potentially directly affect $\mathrm{T}$ cell activation and both favor and hamper antitumor immune responses. Second, the homophilic adhesion molecules CD31 (also known as PECAM1) is expressed on most cells comprising and filling the vascular space but was not yet described on DCs. Several studies report a decrease in expression on leukocytes transmigrating into inflamed tissue or lymph nodes (reviewed by ${ }^{47}$ ) suggesting its absence on mature DCs could be in favor of migration. Finally, we confirmed the maturation induced upregulation of the adhesion molecule JAM-1. Interestingly Jam-1, similar to CCR7 (this paper and ref 32) is efficiently expressed when cells are matured using the clinical grade cytokine cocktail but less expressed by a potent TLR stimulus suggesting that its expression is not associated to DC maturation in all cases. JAM-1 expression is mainly reported on endothelial cells and leukocytes. Like CD31, Jam-1 is able to engage in homotypic interaction but is also a ligand for LFA-1 on leukocytes. ${ }^{48}$ To date there are no reports on Jam-1 expression in human DCs but Jam-1 expression on murine DCs was found negatively associated with migration of DCs to the secondary lymphoid organs. ${ }^{49}$ In conclusion it will be interesting to study the role of all three candidate marker proteins in DC migration and $\mathrm{T}$ cell stimulation and finally to assess how their expression on DCs used for antitumor vaccination relates to clinical outcome.

\section{Conclusions}

We here show that pathway based integration of transcriptome and proteome is a viable approach to develop novel DC maturation markers. Our data demonstrate that the integration of large scale expression data from different sources at the pathway level can be used to increase the predictive power of multi "omics" analysis. We propose this method can serve as an alternative or in supplement to increasing the number of biological replicates.

Abbreviations: DC, dendritic cell; IPI, international protein index, ID, identifier; emPAI, exponentially modified protein abundance index; FET, Fischer exact test; TRS, toll like receptor signaling pathway; CCI, cytokine-cytokine receptor interaction pathway; CAM, cell adhesion molecules pathway; LTM, leukocyte transendothelial migration pathway; PPAR signaling pathway; TLR, toll like receptor.

Acknowledgment. We thank the Microarray Facility Nijmegen for mRNA expression analysis, N. MeeuwsenScharenborg, A. de Boer, and M. van de Rakt for providing DCs. This work was supported by The Netherlands Organization for Scientific Research grant number NWO-9120.6030 and the European network of excellence DC-THERA under the European Commission (EC) Sixth Framework Program project number LSHB-CT-2004-512074.

Supporting Information Available: Tables S1-S8 and Figures S1 and S2. Table S1: Peptide identifications. Detailed lists of peptides and proteins identified from of immature and mature DCs. Table S1a: peptides from immature DCs. Table S1b: peptides from mature DCs. Table S2: Protein expression changes after DC maturation. Detailed list of all identified proteins with emPAI values and ratio's designated "detected" of the protein was detected in both immature DC and mature DC and designated "estimated" if detected in only one condi- 
tion. Table S3: mRNA expression changes after DC maturation. Detailed list of RNA expression ratio's. Table S4: Combined protein and mRNA expression changes after DC maturation. Detailed combined list of RNA and protein data mapped into common entrez IDs. Table S5: Pathway and correlation analysis. Detailed list of results of the pathway and correlation analysis of both proteomics and transcriptomics data using both the Eu.gene analyzer ${ }^{24}$ and the web tool David ${ }^{26,27}$ on all pathways. Table S6: Pathway components. Detailed list of protein and mRNA expression ratio's of all components of the 5 dominant pathways in DC maturation. Table S7: Markers of DC maturation. From Whiteside et al. ${ }^{31}$ a list of 20 well-known DC maturation markers was extracted. Table S8: Enrichement of DC maturation markers in dominant pathways. A set of 20 Dc maturation markers extracted from Whiteside et al. ${ }^{31}$ was by Fischer exact test found significantly enriched in pathways representing dominant DC processes. * affected pathways are those affected by RNA and protein alone or by both (the pathways highlighted in Table 1). Figure S1: The high correlation of pathway components is higher than can be expected as a result of chance. Figure S2: Visual mapping of protein and RNA expression data onto the Kegg "Toll like receptor signaling" pathway. This material is available free of charge via the Internet at http://pubs.acs.org.

\section{References}

(1) Banchereau, J.; Steinman, R. M. Dendritic cells and the control of immunity. Nature 1998, 392 (6673), 245-52.

(2) Guermonprez, P.; Valladeau, J.; Zitvogel, L.; Thery, C.; Amigorena, $\mathrm{S}$. Antigen presentation and T cell stimulation by dendritic cells. Annu. Rev. Immunol. 2002, 20, 621-67.

(3) Steinman, R. M.; Hawiger, D.; Nussenzweig, M. C. Tolerogenic dendritic cells. Annu. Rev. Immunol. 2003, 21, 685-711.

(4) Joffre, O.; Nolte, M. A.; Sporri, R.; Reis e Sousa, C. Inflammatory signals in dendritic cell activation and the induction of adaptive immunity. Immunol. Rev. 2009, 227 (1), 234-47.

(5) Steinman, R. M.; Banchereau, J. Taking dendritic cells into medicine. Nature 2007, 449 (7161), 419-26.

(6) Aarntzen, E. H.; Figdor, C. G.; Adema, G. J.; Punt, C. J.; de Vries, I. J. Dendritic cell vaccination and immune monitoring. Cancer Immunol. Immunother. 2008, 57 (10), 1559-68.

(7) de Vries, I. J.; Lesterhuis, W. J.; Scharenborg, N. M.; Engelen, L. P.; Ruiter, D. J.; Gerritsen, M. J.; Croockewit, S.; Britten, C. M.; Torensma, R.; Adema, G. J.; Figdor, C. G.; Punt, C. J. Maturation of dendritic cells is a prerequisite for inducing immune responses in advanced melanoma patients. Clin. Cancer Res. 2003, 9 (14), 5091-100.

(8) Tang, Z.; Saltzman, A. Understanding human dendritic cell biology through gene profiling. Inflamm. Res. 2004, 53 (9), 424-41.

(9) Manoli, T.; Gretz, N.; Grone, H. J.; Kenzelmann, M.; Eils, R.; Brors, B. Group testing for pathway analysis improves comparability of different microarray datasets. Bioinformatics 2006, 22 (20), 2500-6.

(10) Beltrame, L.; Rizzetto, L.; Paola, R.; Rocca-Serra, P.; Gambineri, L.; Battaglia, C.; Cavalieri, D. Using pathway signatures as means of identifying similarities among microarray experiments. PLoS ONE 2009, 4 (1), e4128.

(11) Le Naour, F.; Hohenkirk, L.; Grolleau, A.; Misek, D. E.; Lescure, P.; Geiger, J. D.; Hanash, S.; Beretta, L. Profiling changes in gene expression during differentiation and maturation of monocytederived dendritic cells using both oligonucleotide microarrays and proteomics. J. Biol. Chem. 2001, 276 (21), 17920-31.

(12) Pereira, S. R.; Faca, V. M.; Gomes, G. G.; Chammas, R.; Fontes, A. M.; Covas, D. T.; Greene, L. J. Changes in the proteomic profile during differentiation and maturation of human monocyte-derived dendritic cells stimulated with granulocyte macrophage colony stimulating factor/interleukin-4 and lipopolysaccharide. Proteomics 2005, 5 (5), 1186-98.

(13) Watarai, H.; Hinohara, A.; Nagafune, J.; Nakayama, T.; Taniguchi, M.; Yamaguchi, Y. Plasma membrane-focused proteomics: dramatic changes in surface expression during the maturation of human dendritic cells. Proteomics 2005, 5 (15), 4001-11.

(14) Horlock, C.; Shakib, F.; Mahdavi, J.; Jones, N. S.; Sewell, H. F.; Ghaemmaghami, A. M. Analysis of proteomic profiles and func- tional properties of human peripheral blood myeloid dendritic cells, monocyte-derived dendritic cells and the dendritic cell-like KG-1 cells reveals distinct characteristics. Genome Biol. 2007, 8 (3), R30.

(15) Gundacker, N. C.; Haudek, V. J.; Wimmer, H.; Slany, A.; Griss, J.; Bochkov, V.; Zielinski, C.; Wagner, O.; Stockl, J.; Gerner, C. Cytoplasmic proteome and secretome profiles of differently stimulated human dendritic cells. J. Proteome Res. 2009, 8 (6), 2799-811.

(16) Jonuleit, H.; Kuhn, U.; Muller, G.; Steinbrink, K.; Paragnik, L.; Schmitt, E.; Knop, J.; Enk, A. H. Pro-inflammatory cytokines and prostaglandins induce maturation of potent immunostimulatory dendritic cells under fetal calf serum-free conditions. Eur. J. Immunol. 1997, 27 (12), 3135-42.

(17) de Vries, I. J.; Eggert, A. A.; Scharenborg, N. M.; Vissers, J. L.; Lesterhuis, W. J.; Boerman, O. C.; Punt, C. J.; Adema, G. J.; Figdor, C. G. Phenotypical and functional characterization of clinical grade dendritic cells. J. Immunother. 2002, 25 (5), 429-38.

(18) Irizarry, R. A.; Hobbs, B.; Collin, F.; Beazer-Barclay, Y. D.; Antonellis, K. J.; Scherf, U.; Speed, T. P. Exploration, normalization, and summaries of high density oligonucleotide array probe level data. Biostatistics 2003, 4 (2), 249-64.

(19) Dai, M.; Wang, P.; Boyd, A. D.; Kostov, G.; Athey, B.; Jones, E. G.; Bunney, W. E.; Myers, R. M.; Speed, T. P.; Akil, H.; Watson, S. J.; Meng, F. Evolving gene/transcript definitions significantly alter the interpretation of GeneChip data. Nucleic Acids Res. 2005, 33 (20), e175.

(20) Lasonder, E.; Ishihama, Y.; Andersen, J. S.; Vermunt, A. M.; Pain, A.; Sauerwein, R. W.; Eling, W. M.; Hall, N.; Waters, A. P.; Stunnenberg, H. G.; Mann, M. Analysis of the Plasmodium falciparum proteome by high-accuracy mass spectrometry. Nature 2002, 419 (6906), 537-42.

(21) Rappsilber, J.; Ishihama, Y.; Mann, M. Stop and go extraction tips for matrix-assisted laser desorption/ionization, nanoelectrospray, and LC/MS sample pretreatment in proteomics. Anal. Chem. 2003, 75 (3), 663-70.

(22) Ishihama, Y.; Oda, Y.; Tabata, T.; Sato, T.; Nagasu, T.; Rappsilber, J.; Mann, M. Exponentially modified protein abundance index (emPAI) for estimation of absolute protein amount in proteomics by the number of sequenced peptides per protein. Mol. Cell. Proteomics 2005, 4 (9), 1265-72.

(23) Ishihama, Y.; Schmidt, T.; Rappsilber, J.; Mann, M.; Hartl, F. U.; Kerner, M. J.; Frishman, D. Protein abundance profiling of the Escherichia coli cytosol. BMC Genomics 2008, 9, 102.

(24) Cavalieri, D.; Castagnini, C.; Toti, S.; Maciag, K.; Kelder, T.; Gambineri, L.; Angioli, S.; Dolara, P. Eu.Gene Analyzer a tool for integrating gene expression data with pathway databases. Bioinformatics 2007, 23 (19), 2631-2.

(25) Grosu, P.; Townsend, J. P.; Hartl, D. L.; Cavalieri, D. Pathway Processor: a tool for integrating whole-genome expression results into metabolic networks. Genome Res. 2002, 12 (7), 1121-6.

(26) Huang da, W.; Sherman, B. T.; Lempicki, R. A. Systematic and integrative analysis of large gene lists using DAVID bioinformatics resources. Nat. Protoc. 2009, 4 (1), 44-57.

(27) Dennis, G., Jr.; Sherman, B. T.; Hosack, D. A.; Yang, J.; Gao, W.; Lane, H. C.; Lempicki, R. A. DAVID: Database for Annotation, Visualization, and Integrated Discovery. Genome Biol. 2003, 4 (5), P3.

(28) Segota, I.; Bartonicek, N.; Vlahovicek, K. MADNet: microarray database network web server. Nucleic Acids Res. 2008, 36 (Web Server issue), W332-5.

(29) Benjamini, Y.; Drai, D.; Elmer, G.; Kafkafi, N.; Golani, I. Controlling the false discovery rate in behavior genetics research. Behav. Brain Res. 2001, 125 (1-2), 279-84.

(30) Mathivanan, S.; Ahmed, M.; Ahn, N. G.; Alexandre, H.; Amanchy, R.; Andrews, P. C.; Bader, J. S.; Balgley, B. M.; Bantscheff, M.; Bennett, K. L.; Bjorling, E.; Blagoev, B.; Bose, R.; Brahmachari, S. K.; Burlingame, A. S.; Bustelo, X. R.; Cagney, G.; Cantin, G. T.; Cardasis, H. L.; Celis, J. E.; Chaerkady, R.; Chu, F.; Cole, P. A.; Costello, C. E.; Cotter, R. J.; Crockett, D.; DeLany, J. P.; De Marzo, A. M.; DeSouza, L. V.; Deutsch, E. W.; Dransfield, E.; Drewes, G.; Droit, A.; Dunn, M. J.; Elenitoba-Johnson, K.; Ewing, R. M.; Van Eyk, J.; Faca, V.; Falkner, J.; Fang, X.; Fenselau, C.; Figeys, D.; Gagne, P.; Gelfi, C.; Gevaert, K.; Gimble, J. M.; Gnad, F.; Goel, R.; Gromov, P.; Hanash, S. M.; Hancock, W. S.; Harsha, H. C.; Hart, G.; Hays, F.; He, F.; Hebbar, P.; Helsens, K.; Hermeking, H.; Hide, W.; Hjerno, K.; Hochstrasser, D. F.; Hofmann, O.; Horn, D. M.; Hruban, R. H.; Ibarrola, N.; James, P.; Jensen, O. N.; Jensen, P. H.; Jung, P.; Kandasamy, K.; Kheterpal, I.; Kikuno, R. F.; Korf, U.; Korner, R.; Kuster, B.; Kwon, M. S.; Lee, H. J.; Lee, Y. J.; Lefevre, M.; Lehvaslaiho, M.; Lescuyer, P.; Levander, F.; Lim, M. S.; Lobke, C.; 
Loo, J. A.; Mann, M.; Martens, L.; Martinez-Heredia, J.; McComb, M.; McRedmond, J.; Mehrle, A.; Menon, R.; Miller, C. A.; Mischak, H.; Mohan, S. S.; Mohmood, R.; Molina, H.; Moran, M. F.; Morgan, J. D.; Moritz, R.; Morzel, M.; Muddiman, D. C.; Nalli, A.; Navarro, J. D.; Neubert, T. A.; Ohara, O.; Oliva, R.; Omenn, G. S.; Oyama M.; Paik, Y. K.; Pennington, K.; Pepperkok, R.; Periaswamy, B.; Petricoin, E. F.; Poirier, G. G.; Prasad, T. S.; Purvine, S. O.; Rahiman, B. A.; Ramachandran, P.; Ramachandra, Y. L.; Rice, R. H.; Rick, J.; Ronnholm, R. H.; Salonen, J.; Sanchez, J. C.; Sayd, T.; Seshi, B.; Shankari, K.; Sheng, S. J.; Shetty, V.; Shivakumar, K.; Simpson, R. J.; Sirdeshmukh, R.; Siu, K. W.; Smith, J. C.; Smith, R. D.; States, D. J.; Sugano, S.; Sullivan, M.; Superti-Furga, G.; Takatalo, M.; Thongboonkerd, V.; Trinidad, J. C.; Uhlen, M.; Vandekerckhove, J.; Vasilescu, J.; Veenstra, T. D.; VidalTaboada, J. M.; Vihinen, M.; Wait, R.; Wang, X.; Wiemann, S.; Wu, B.; Xu, T.; Yates, J. R.; Zhong, J.; Zhou, M.; Zhu, Y.; Zurbig, P.; Pandey, A. Human Proteinpedia enables sharing of human protein data. Nat. Biotechnol. 2008, 26 (2), 164-7.

(31) Whiteside, T. L.; Odoux, C. Dendritic cell biology and cancer therapy. Cancer Immunol. Immunother. 2004, 53 (3), 240-8.

(32) Lehner, M.; Morhart, P.; Stilper, A.; Petermann, D.; Weller, P.; Stachel, D.; Holter, W. Efficient chemokine-dependent migration and primary and secondary IL-12 secretion by human dendritic cells stimulated through Toll-like receptors. J. Immunother. 2007, 30 (3), 312-22.

(33) Waters, K. M.; Pounds, J. G.; Thrall, B. D. Data merging for integrated microarray and proteomic analysis. Brief Funct. Genomic Proteomic 2006, 5 (4), 261-72.

(34) Randolph, G. J.; Ochando, J.; Partida-Sanchez, S. Migration of dendritic cell subsets and their precursors. Annu. Rev. Immunol. 2008, 26, 293-316.

(35) Tontonoz, P.; Spiegelman, B. M. Fat and beyond: the diverse biology of PPARgamma. Annu. Rev. Biochem. 2008, 77, 289-312.

(36) Nencioni, A.; Grunebach, F.; Zobywlaski, A.; Denzlinger, C.; Brugger, W.; Brossart, P. Dendritic cell immunogenicity is regulated by peroxisome proliferator-activated receptor gamma. J. Immunol. 2002, 169 (3), 1228-35.

(37) Szatmari, I.; Gogolak, P.; Im, J. S.; Dezso, B.; Rajnavolgyi, E.; Nagy, L. Activation of PPARgamma specifies a dendritic cell subtype capable of enhanced induction of iNKT cell expansion. Immunity 2004, 21 (1), 95-106.

(38) Szatmari, I.; Nagy, L. Nuclear receptor signalling in dendritic cells connects lipids, the genome and immune function. Embo J. 2008, 27 (18), 2353-62.
(39) Varga, T.; Nagy, L. Nuclear receptors, transcription factors linking lipid metabolism and immunity: the case of peroxisome proliferator-activated receptor gamma. Eur. J. Clin. Invest. 2008, 38 (10), 695-707.

(40) Szatmari, I.; Torocsik, D.; Agostini, M.; Nagy, T.; Gurnell, M.; Barta, E.; Chatterjee, K.; Nagy, L. PPARgamma regulates the function of human dendritic cells primarily by altering lipid metabolism. Blood 2007, 110 (9), 3271-80.

(41) Ye, J. Regulation of PPARgamma function by TNF-alpha. Biochem. Biophys. Res. Commun. 2008, 374 (3), 405-8.

(42) Selenko-Gebauer, N.; Majdic, O.; Szekeres, A.; Hofler, G.; Guthann, E.; Korthauer, U.; Zlabinger, G.; Steinberger, P.; Pickl, W. F.; Stockinger, H.; Knapp, W.; Stockl, J. B7-H1 (programmed death-1 ligand) on dendritic cells is involved in the induction and maintenance of T cell anergy. J. Immunol. 2003, 170 (7), 3637-44.

(43) Keir, M. E.; Butte, M. J.; Freeman, G. J.; Sharpe, A. H. PD-1 and its ligands in tolerance and immunity. Annu. Rev. Immunol. 2008, 26, 677-704.

(44) Sharpe, A. H.; Wherry, E. J.; Ahmed, R.; Freeman, G. J. The function of programmed cell death 1 and its ligands in regulating autoimmunity and infection. Nat. Immunol. 2007, 8 (3), 239-45.

(45) Brown, J. A.; Dorfman, D. M.; Ma, F. R.; Sullivan, E. L.; Munoz, O.; Wood, C. R.; Greenfield, E. A.; Freeman, G. J. Blockade of programmed death-1 ligands on dendritic cells enhances $\mathrm{T}$ cell activation and cytokine production. J. Immunol. 2003, 170 (3), 1257-66.

(46) Dong, H.; Zhu, G.; Tamada, K.; Chen, L. B7-H1, a third member of the B7 family, co-stimulates T-cell proliferation and interleukin10 secretion. Nat. Med. 1999, 5 (12), 1365-9.

(47) Woodfin, A.; Voisin, M. B.; Nourshargh, S. PECAM-1: a multifunctional molecule in inflammation and vascular biology. Arterioscler. Thromb. Vasc. Biol. 2007, 27 (12), 2514-23.

(48) Ostermann, G.; Weber, K. S.; Zernecke, A.; Schroder, A.; Weber, C. JAM-1 is a ligand of the beta(2) integrin LFA-1 involved in transendothelial migration of leukocytes. Nat. Immunol. 2002, 3 (2), 151-8.

(49) Cera, M. R.; Del Prete, A.; Vecchi, A.; Corada, M.; Martin-Padura, I.; Motoike, T.; Tonetti, P.; Bazzoni, G.; Vermi, W.; Gentili, F.; Bernasconi, S.; Sato, T. N.; Mantovani, A.; Dejana, E. Increased DC trafficking to lymph nodes and contact hypersensitivity in junctional adhesion molecule-A-deficient mice. J. Clin. Invest. 2004, 114 (5), 729-38.

PR9008546 